\title{
La culture dense de Pasteurella multocida méthode de choix pour la production du vaccin contre la Pasteurellose bovine
}

\author{
por P. PERREAU
}

\section{INTRODUCTION}

L'obtention de cultures microbiennes denses, par aération et agitation des milieux, n'est plus une méthode neuve, mais il semble qu'elle est peu utilisée, bien moins qu'elle devrait l'être à notre avis ; la boîte de Roux constitue encore dans de nombreux laboratoires le matériel de base pour la production des vaccins bactériens.

II est certain que la méthode ne s'adresse en principe qu'aux laboratoires ou instituts qui préparent de grosses quantités d'antigènes ou de toxines destinés essentiellement à la vente comme vaccins, et secondairement aux recherches de physiologie microbienne ou d'immunologie, ce qui limite donc obligatoirement le nombre de gens intéressés par ces techniques.

En outre, elle ne paraît pas se répandre autant qu'on pourrait l'espérer, car, aux yeux de bien des bactériologistes et de façon injustifiée, elle reste l'apanage des spécialistes et requiert un matériel compliqué et coûteux.

Aussi voudrions-nous apporter, dans cette note. d'une part notre contribution au développement d'une méthode extrêmement intéressante, que nous avons appliquée personnellement à la production de vaccin contre la pasteurellose bovine («Pasteurella multocida » type I de ROBERTS ou $B$ de (ARTER), d'autre part une réponse à une recommandation faite à la conférence $F$. A. O. de MANILLE (Philippines) en 1959 et concernant l'influence des agents antimoussants sur la croissance et la capsulogenèse de $P$. multocida.

La culture en milieu aéré ef agité, qu'elle soit continue ou en masse, a fait l'objet de divers travaux tant théoriques que pratiques depuis

Reçu pour publication : mai 1961.

Rev. Elev. Méd. vét. Pays trop., 1961, 14, no 2. une douzaine d'années; aussi nous permettrons nous un bref rappel des études les plus importantantes.

\section{HISTORIQUE}

En 1950, MONOD (5) établit la théorie de la technique de culture continue et évoque les applications qu'elle serait susceptible de recevoir tandis que NOVICK et SZILARD (7) décrivent leur «Chemostat».

En 1952, CHAIN et ses collaborateurs décrivent un procćdé d'aération d'un milieu par tourbillon.

Van DRIMMELEN (12) en 1956, au laboratoire d'Onderstepoort, cultive avec succès la souche Brucella abortus 19 en flacon agité, tandis que NEWING et MAC LEOD (6) rapportent leurs expériences faites sur de petits volumes de milieu agités par tourbillon sur agitateur magnétique et obtiennent des cultures très riches de Pasteurella multocida et de Mycoplasma mycoides.

En 1958, Van DRIMMELEN (13) utilise la culture en masse aérée par tourbillon pour préparer le vaccin B-19 contre la brucellose bovine, avec de bons résultats.

Cette même année, la production de cultures très denses de Pasteurella multocida est obtenue d'une part par STERNE ef HUTCHINSON (10) qui aèrent leur milieu par pulvérisation de fines bulles d'air, d'autre part par BAIN et JONES (2) qui emploient le procédé désormais elassique de l'aération par tourbillon.

Toujours en 1958. STERNE (9) décrit une technique de culture de Brucella abortus 19 en fube de cellophane aéré, qui fournit des récoltes fitrant jusqu'à $50-70 \mathrm{~g}$ de poids sec de bactéries par litre.

WEYLAND (14) en 1960 passe en revue tous 
les avantages que présente la méthode de culture continue et, à l'aide de quelques exemples, souligne tout l'intérêt que nous devons lui attacher.

\section{MÉTHODE}

\section{Io Matériel}

Notre choix s'est porté sur un appareil non spécialisé, pouvant servir à diverses productions, en l'occurrence un fermentateur fabriqué par la firme Terzano ef Cie de Milan (modèle TB-25). (voir figure 1).

Il s'agit là d'un appareil de conception très classique, identique à tous les fermentateurs de petit volume utilisés dans les essais de production d'antibiotiques.

C'est une cuve en pyrex, d'un volume utile de 25 litres environ, stérilisable soit à l'autoclave soit par injection de vapeur, un raccordement par tube flexible pouvant se faire sur une chaudière génératrice de vapeur.

Cette cuve est fermée par un plateau en acier inoxydable laissant passer par plusieurs orifices les accessoires indispensables :

- l'axe de la turbine, monté sur roulements et entraîné par un moteur électrique au moyen d'une courroie ; les deux poulies du raccordement moteur-axe de la turbine présentent chacune trois gorges, ce qui permet d'obtenir une gamme de vitesses de rotation allant de 300 à 600 tours/minute. Le diamètre de la turbine à 8 pales est de $74 \mathrm{~mm}$ pour un diamètre de la cuve de $280 \mathrm{~mm}$.

- un tube d'insuflation d'air qui peut être soit très court, l'air est alors envoyé à la surface du milieu, soit long ef débouchant sous la turbine, l'air est alors injecté en profondeur ef les bulles sont pulvérisées par les pales de celle-ci.

- trois appareils assurant la régulation thermique dans le milieu de culture : une bougie chauffante, une bougie réfrigérante et un thermomètre à contact réglable couplé avec un relais électronique.

Ce fermentateur est en effet complètement autonome quant à son chauffage; il n'a besoin ni de bain-marie, ni d'étude et peut s'installer n'importe où dans un laboratoire.

La précision du contrôle de la température est remarquable ef l'amplitude des oscillations thermométriques très faibles.
- deux orifices équipés de presse-étoupe par lesquels on fait entrer dans l'appareil des tubulures diverses destinées aux prélèvements d'échantillon ou à la récolte ; ils peuvent aussi être munis de disques en caoutchouc perforables au travers desquels une aiguille sert à introduire à tout moment des substances telles que sérum, solution tampon, sucre, facteur de croissance, anti-mousse, etc...

Le plateau d'acier qui supporte cet appareillage s'applique de façon étanche à la cuve de pyrex grâce à un joint circulaire, le serrage s'effectuant par 6 vis à ailettes.

L'ensemble plateau-cuve est encastré dans un trépied qui maintient la rigidité du tout.

Ce fermentateur peut donc se prêter à diverses modifications; : selon les impératifs des cultures envisagées; nous l'avons toujours fait fonctionner sans bougie réfrigérante, la faible inertie du système thermomètre-bougie chauffante la rendant inutile dans nos conditions de travail; les oscillations thermométriques n'ont ainsi jamais atteint $0,2^{\circ} \mathrm{C}$.

L'air envoyé dans le fermentateur est fourni par un compresseur Marion d'un débit de 25 litres/heure à la pression atmosphérique; injecté au travers d'un filtre en verre fritté de grosse porosité ( $n^{0} 1$ ), il barbote dans un flacon laveur empli d'eau, passe dans un second flacon où les gouttelettes projetées par la turbulence gazeuse se déposent et traverse ensuite un filtre Seitz de $100 \mathrm{ml}$ (disque EKS $\varnothing 6 \mathrm{~cm}$ ) oì sa stérilité est acquise. Ce procédé nous a donné des résultats excellents et nous l'avons adopté définitivement, convaincu de sa parfaite efficacité.

Le milieu est réparti en flacons. Pyrex de 10 litres à tubulure inférieure équipée d'un tube muni d'une forte aiguille protégée, par laquelle le bouillon pourra s'écouler dans le fermentateur au travers de la membrane de caoutchouc (Voir figure no 2).

Surle tube d'écoulement est intercalé un comptegouttes à transfusion servant à contrôler le débit avec l'aide d'une pince presse-tube à vis.

\section{Souches}

Les souches de «Pasteurella multocida » utilisées au cours de ces essais de production sont toutes des souches du type I de ROBERTS isolées dans des foyers de septicémie hémorragique 


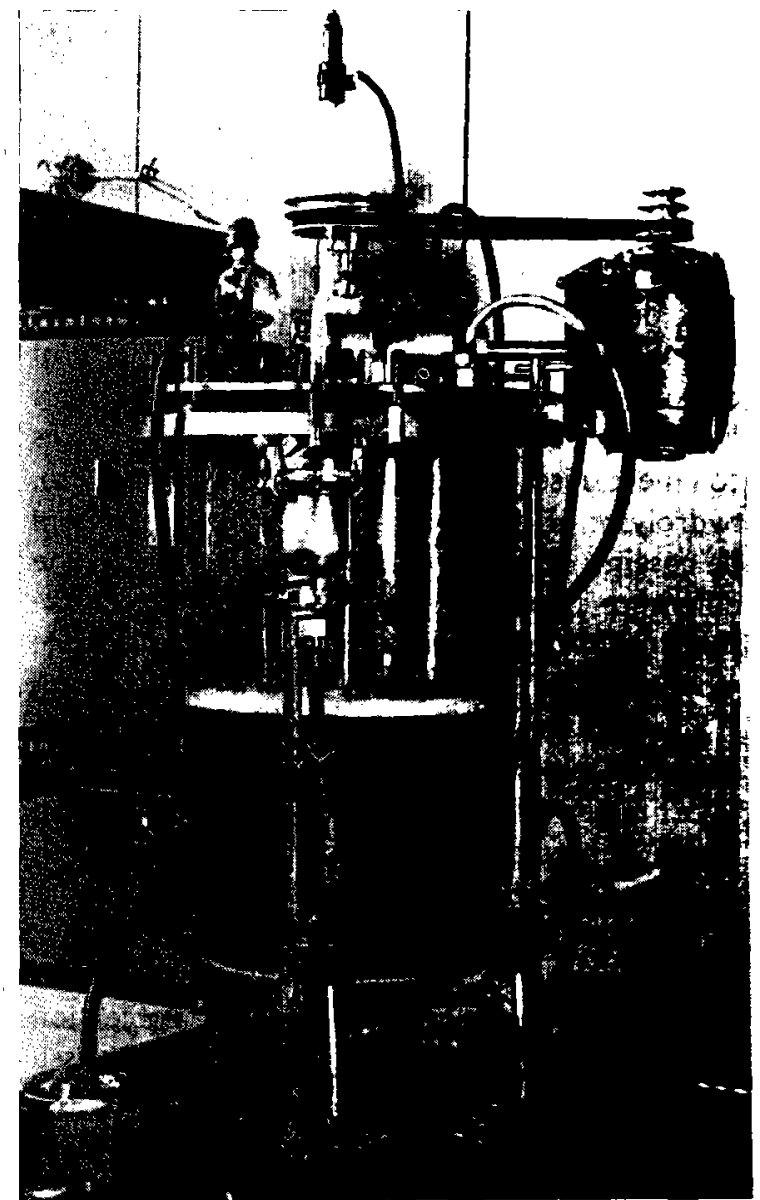

Fig. 1. - Appareil utilisé (fermenteur Terzano).

soit chez des zébus en Afrique Centrale, soif chez des buffles en Asie.

Toutes nous ont donné des récoltes comparables dans des conditions de cultures identiques.

\section{3o Milieux}

Un certain nombre de milieux furent ufilisés, mais c'est principalement le milieu décrit par STERNE (10) que nous avons employé dont la caractéristique essentielle est de contenir en quantité notable un autodigestat de pancréas de bœuf.

Nous reviendrons plus loin sur les résultats que nous ont fourni les différents milieux.

\section{Culture}

Le fermentateur est stérilisé tout monté, à l'autoclave durant deux heures (90 minutes à

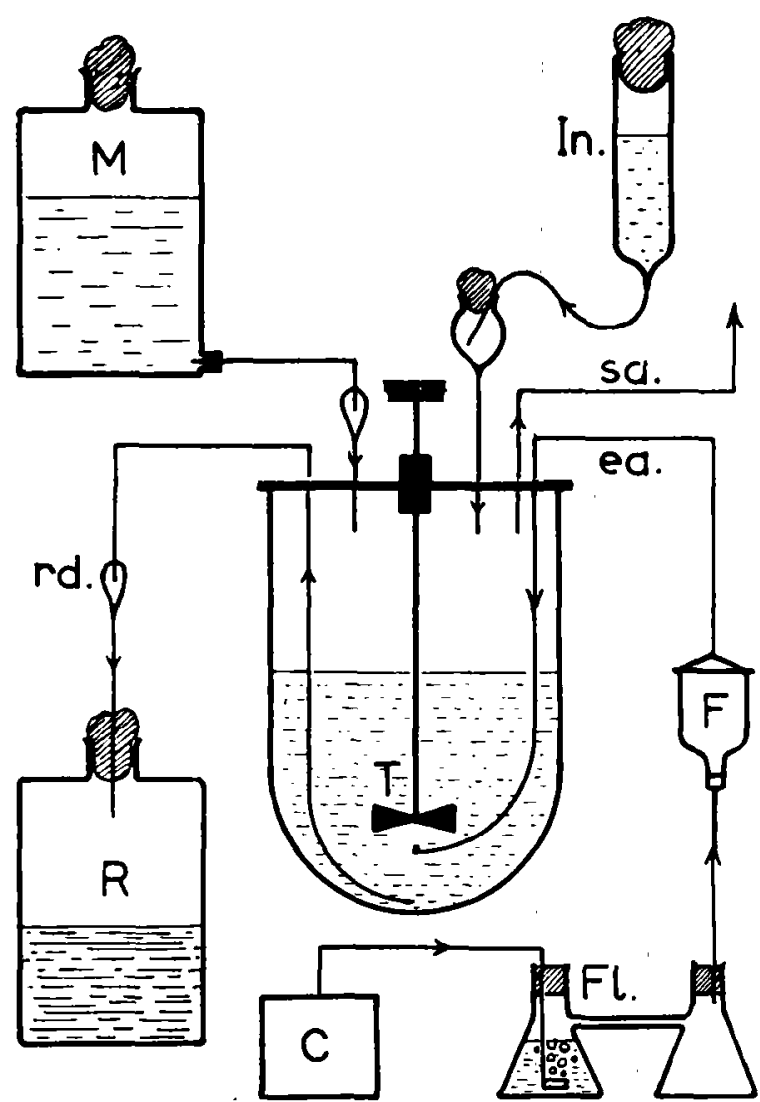

FIG. 2. - DIAGRAMME DE FONCTIONNEMENT

C : Compresseur ; F : Filtre Seitz ; FI : Flacon barboleur ; In : Inoculum ; $M$ : Réserve de milieu ; $R$ : Récolte : T: Turbine ; ea : Entrée d'air ; sa : Sortie d'air ; rd : Régulateur de débit (à transfusion).

$110^{\circ}$ et 30 minutes à $\left.115^{\circ}\right)$; ce procédé s'est révélé plus commode que la stérilisation par injection de vapeur à l'intérieur.

La cuve de pyrex reçoit 15 litres de milieu et le chauffage est mis en marche; une fois la température stabilisée à $37^{\circ}$, ce qui demande environ 30 minutes, 200 à $400 \mathrm{ml}$ d'une culture en bouillon de 6 à 8 heures sont introduits.

En règle constante, nous n'avons utilisé que des hémocultures jeunes faites en bouillon au tryptose avec du sang virulent de lapin.

Cet inoculum est contenu dans une allonge de $500 \mathrm{ml}$ dont la tubulure inférieure est munie, par l'intermédiaire d'un tube de caoutchouc, d'une effilure de pipette : lors de l'ensemencement celle-ci est brisée dans la flamme, engagée dans le col du ballon « ad hoc », où elle est serrée 
par le tampon de gaze. I. a manipulation est simple et sa stérilité est assurée.

Quelques instants avant, ont été introduits également par la même voie l'anti-mousse, les solutions de phosphates, le sang lysé, etc... selon le milieu employé.

La turbine est mise en route aussitôt, car dans cet appareil, elle assure non seulement l'aération du milieu, mais l'homogénéisation de la température dans toute la masse liquide.

L'air n'est envoyé qu'ultérieurement au bout de 2 à 3 heures en général. La croissance microbienne s'accélère rapidement dès la $5 \mathrm{e}$ heure et entre dans sa phase logarithmique; le plafond est atteint dès la 10e-12e heure de culture; ensuite celle-ci se poursuit soit en masse, soit en fonctionnement continu, ce qui permet d'augmenter encore la densité optique de la suspension.

\section{RÉSULTATS ET COMMENTAIRES}

Les suspensions récoltées au cours de notre. production avaient une opacité variant entre le tube no 20 ef le tube no 35 de BROWN; la période de mise au point étant terminée, la densité optique de nos récoltes actuelles n'est jamais inférieure à celle du tube no 24 , pourvu qu'un certain nombre de conditions que nous allons maintenant préciser et discuter soient remplies.

\section{Io Milieux de culture :}

Le milieu qui nous a fourni les meilleures récoltes ef que nous utilisons maintenant est celui décrit par STERNE et HUTCHINSON (10).

Nous avons passé en revue, au cours de nos essais, un certain nombre de milieux, en allant des plus simples vers les plus complexes.

Les milieux ordinaires peptonés, simplement enrichis par de l'extrait de levure, du sérum ou du sang lysé de bœuf n'ont fourni que des récoltes de faible densité optique (tube 3 à 5 de BROWN).

Les milieux dans lesquels l'azote était apporté sous forme d'hydrolysats trypsiques de caséine (Tryptone Oxoïd, Bacto-Tryptone et Bacto-Tryptose Dif(co) se sont révélés nettement meilleurs que les premiers, mais encore insuffisants pour obtenir de réelles cultures denses.

Nous avons ensuite employé le milieu utilisé par BAIN et JONES (2), pensant qu'il allait nous donner d'excellents résultats étant donné que notre appareil avec son aération par tourbillon est pratiquement identique au «fût à bière » décrit par ces deux auteurs; or les résultats ont été décevants et nous ne savons pas encore pourquoi.

Ce milieu a été préparé en suivant les indications de BAIN, à cette différence près que nous n'avons pas préparé nous-même l'hydrolysat acide de caséine et l'extrait de levure; nous avons utilisé successivement trois hydrolysats commercialisés: Bacto-casamino Acid's Difco, hydrolysat acide de caséine Oxoid et hydrolysat de caséine déchloruré Liebig, sans constater une différence appréciable dans les résultats qui se sont traduits dans ces cultures par une opacité moyenne égale à celle du tube no 6 de BROWN.

L'extrait de levure utilisé était le Yeast Extract Difco aux taux de 2,5 et $5 \mathrm{~g}$ par litre.

Le milieu de STERNE par contre nous a donné de très bons résultats et l'autodigestat de pancréas de bœuf se confirme être un puissant stimulant de la croissance de $P$. multocida.

Le digestat papaïnique de pancréas se montre d'une valeur comparable et permet l'obtention de cultures aussi denses. L'autodigestion du tissu pancréatique ne semble d'ailleurs pas libérer la totalité du ou des facteurs de croissance qu'il contient, comme nous l'a démontré l'expérience suivante: plusieurs kilos de pancréas sont hachés et autodigérés selon la méthode de STERNE *, et le filtrat nous permet d'obtenir dans une première culture 40 litres environ de suspension microbienne à opacité no 20 de BROWN; le résidu retenu par le filtre est alors digéré complètement par la papainne ef sert après filtration à préparer à nouveau 25 litres de milieu, en étant employé comme substitut du premier filtrat; une densité optique égale au tube no 13 de BROWN est alors atteinte.

Il serait donc intéressant de pouvoir extraire complètement du pancréas de bœuf le ou les facteurs de croissance, par une méthode moins empirique que la simple autodigestion.

Au cours de plusieurs essais, nous avons substitué le foie de bœuf au pancréas soit en employant une infusion de foie comme milieu de base, soit

* Nous remercions vivement le Dr STERNE de nous avoir aimablement communiqué sa recette de digestion pancréatique. 
en remplaçant dans le milieu de STERNEl'autodigestat pancréatique par une quantité équivalente de digestion papaïnique de foie ; les résultats ont toujours été inférieurs à ceux fournis par le milieu original.

L'addition à ces milieux de chlorhydrate de thiamine, de nicotinamide et de pantothénate de sodium n'a eu aucune influence sur la croissance, ce qu'avait déjà observé BAIN (1).

\section{Régulation du $\mathrm{pH}$ :}

Dans le milieu de BAIN, qui est tamponné par des phosphates, le $\mathrm{pH}$ n'est jamais descendu dans nos essais au-dessous de 6,5; avec le milieu de STERNE, le pH observé à son point le plus bas au cours d'une culture est de 6,3 (il faut noter qu'ici l'apport de milieu neuf en culture continue joue un très grand rôle dans le maintien d'un $\mathrm{pH}$ convenable), et de façon constante, qu'il s'agisse de culture en masse (discontinue) ou de culture continue avec apport insuffisant de milieu neuf, on observe après l'acidification initiale une alcalinisation progressive du milieu avec remontée $\mathrm{du} \mathrm{pH}$ à des valeurs supérieures à 8 .

\section{3o Aération :}

Le volume d'air insuflé dans notre fermentateur peut varier dans des proportions assez fortes sans que l'on constate de variations significatives dans la richesse de la culture.

Le débit mesuré après le passage dans le filtre SEITZ par un ajustage de VENTURI et un manomètre est d'environ $350 \mathrm{ml}$ par minute et par litre de milieu; il est donc supérieur à celui qui est indiqué pour les appareils de BAIN et de STERNE et qui est d'environ $200 \mathrm{ml}$ par minute et par litre de milieu.

Mais avec un même milieu et pour un débit d'air égal, la richesse de la culture varie considérablement selon le mode d'insuflation dans l'appareil : avec l'aération en surface (type BAIN), c'est-à-dire sans barbotage, la culture est pauvre, tandis qu'elle est très riche avec l'aération en profondeur, c'est-à-dire avec barbotage et pulvérisation des bulles par la turbine.

L'expérience, répétée plusieurs fois, donne pour le même lot de milieu de STERNE des densités optiques qussi inégales que les tubes 6 et 30 de BROWN selon le mode d'insuflation, toutes les autres conditions de cultures étant rigoureusement identiques ; cette constatation semble contredire les observations de BAIN et de STERNE selon lesquelles le mode d'aération n'a que peu d'importance par rapport à la composition du milieu, mais il se peut que le « vortex » de notre appareil soit moins efficace que celui de l'appareil de BAIN pour de simples différences de vitesse, de diamètre de la turbine ou même de forme du récipient, ce qui est loin d'être évident cependant.

A notre avis l'aération dont il s'agit est beaucoup plus qu'une simple oxygénation du milieu, c'est autant une sorte de «lavage gazeux » de la culture entraînant avec lui de nombreux produits volatils du métabolisme.

Dans notre fermentateur, l'aération en surface peut donc être considérée comme insuffisante pour la seule raison qu'elle se comporte en facteur limitant.

\section{$4^{\circ}$ Volume de l'inoculum:}

Celui-ci doit être copieux et effcctuć avec une culfure de 12 heures au plus.

Pour les 15 litres de milieu habituellement contenus dans le fermentateur, nous n'employons plus d'inoculum dont le volume est inférieur à 300 à $400 \mathrm{ml}$. Plus l'inoculum est copieux, plus la phase de latence de la culture est faible et plus les chances d'obtenir une forte densité sont acquises.

Avec $400 \mathrm{ml}$ d'une hémoculture introduite dar:s 15 litres de milieu de STERNE, nous avons obtenu en 6 heures la densité optique du tube no 35 de BROWN.

\section{Contaminations :}

Elles ne sont nullement à craindre si l'on opère selon les règles classiques des manipulations bactériologiques.

Notre appareil a fonctionné deux jours par semaine quatre mois durant sans le moindre accident de contamination.

Le risque n'existe d'ailleurs vraiment qu'à l'ensemencement; en cours de culture, lors d'addition de sérum, d'antimousse ou de produits divers, la masse de bactéries en multiplication est telle qu'un petit nombre de germes contaminants introduits accidentellement n'a pratiquement aucune chance de résister à la sélection et est rapidement éliminé. 
Nous ne pouvons donc qu'approuver pleinement ce qu'a déjà écrit STERNE (11) sur l'importance de ce risque, en insistant cependant sur les trois conditions qui sont d̀ nos yeux capitales:

1) le contrôle rigoureux des stérilisations de l'appareil, des milieux, des filtres ef autres accessoires.

2) la légère surpression ( 8 d̀ $15 \mathrm{~cm}$ d'eau) qui règne en permanence dans le fermentateur pendant la culture:

3) la rigueur microbiologique des manipulations qui se font toutes dans la flamme.

\section{$6^{0}$ Action de l'antimousse :}

Alors que dans l'aération par simple tourbilIon l'emploi d'un antimousse n'est guère nécessaire, celui-ci devient indispensable lorsque

TABLEAU I - Titrage de l'antigène capsulaire

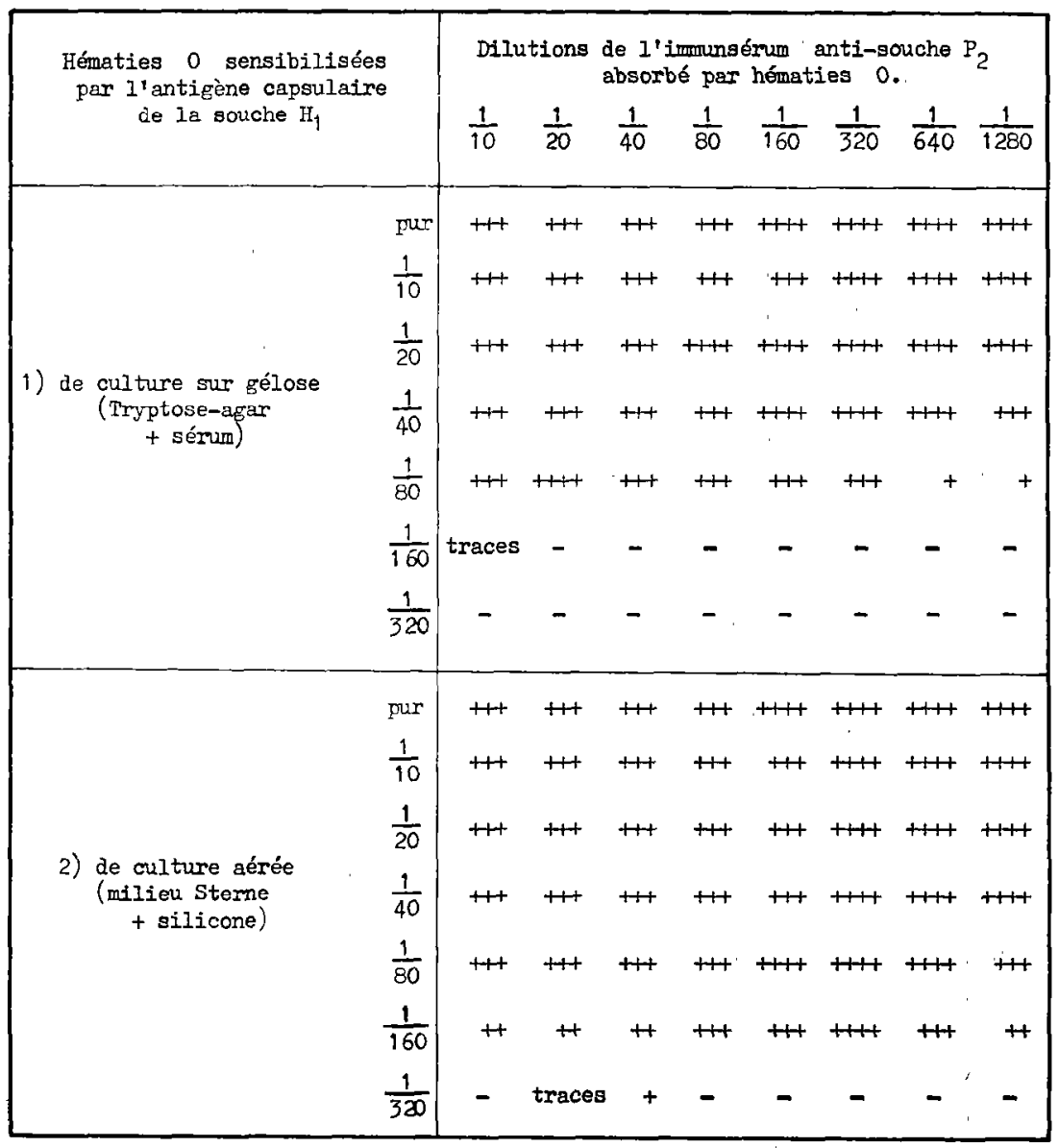

- Dans cette expérience, la souche $\mathrm{H}_{1}$ de Pasteurella multocida sert à préparer, à partir de la même hémoculture, deux récoltes microbiennes, l'une sur gélose, l'autre en bouillon aéré contenant l'antimousse Rhodorsil 426.

- Les deux récoltes ajustées à l'opacité no 35 de Brown, traitées par la méthode de Carter (déplacement par la chaleur de l'antigène capsulaire) fournissent après centrifugation un matériel antigénique qui est adsorbé sur des hématies humaines.

- Les souches $\mathrm{H}_{1}$ ef $\mathrm{P}_{2}$ sont du même groupe (B de Carter ou plus exactement du sous-groupe $B$ africain).

- L'anti-sérum détecte encore très nettement l'antigène « siliconé » dilué au $1 / 160$ alors qu'à cette même dilution l'antigène de culture sur gélose n'est plus décelable. relliques.

- Remarquer le phénomène de zone, très courant dans l'hémagglutination passive avec les sérums anti-pasteu- 
l'air est pulvérisé dans la masse liquide, a fortiori si celle-ci contient du sang lysé ou du sérum.

Nous avons utilisé de façon constante l'antimousse Rhodorsil 426 (émulsion aqueuse à $30 \mathrm{p}$. 100 de silicone) à des concentrations de 1 et $2 p$. 1.000 sans que la croissance des germes en soit affectée.

Des essais préliminaires en milieu stagnant avaient montré que des concentrations variant de 0,5 p. 1.000 à 50 p. 1.000 n'entraînaient aucune inhibition de la culture.

L'antimousse est ajouté dans le bouillon le plus souvent par fractions de 10 ou $20 \mathrm{ml}$ seion le débit du milieu et selon le degré de moussage de celui-ci ; en général une dose de $20 \mathrm{ml}$ prévient fout moussage dangereux dans les 12 heures qui suivent.

BAIN (2) a signalé un freinage relatif des culfures par addition d'antimousse; d'autre part, à la Conférence FAO-OIE de MANILLE (8), en décembre 1959, la question fut soulevée de savoir si les agents anti-moussants n'entravaient pas la capsulogenèse de Pasteurella multocida, influence qui serait fort gênante car l'antigène capsulaire est un élément important des antigènes vaccinants.

Dans nos conditions de travail et avec le Rhodorsil 426, nous n'avons jamais observé d'inhibition relative de la culture ou d'absence de capsulogenèse.

Celle-ci est visible à l'examen en contraste de phase et sur les préparations faites à l'encre de Chine tout autant qu'avec les germes récoites sur gélose.

En utilisant la méthode préconisée par CARTER (3) nous avons d'ailleurs préparé souvent un antigène capsulaire de type $B$ à partir de notre récolte en bouillon siliconé. Les hématies $O$ sensibilisées par cet antigène sont agglutinées par un immunsérum aux mêmes titres que les hématies sensibilisées par un antigène préparé avec des cultures sur gélose; en outre, si des dilutions progressives de ces deux types antigènes sont adsorbées sur les hématies, c'est pour la même dilution antigénique que l'hémagglutination devient négative (ou tout au moins pour une dilution très voisine), ce qui montre que dans les deux modes de culture l'élaboration de l'antigène spécifique capsulaire est comparable (voir tableau I).

\section{CONCLUSIONS}

La culture dense de Pasteurella multocida est à la portée de tous les laboratoires et elle est indispensable à notre avis à tous ceux qui préparent de grosses quantités de vaccin contre les pasteurelloses animales, car elle permet un gain de temps considérable et évite des manipulations fastidieuses. Elle ne requiert pas un matériel étroitement spécialisé et l'on peut trouver aujourd'hui dans de nombreux pays des appareils comparables à notre fermentateur qui, crec quelques adaptations ou modifications mineures, permettront d'obtenir d'excellents résultats en partant des travaux de base que sont les expérimentations réalisées d'une part par BAIN ef JONES (2), d'autre part par STERNE et HUTCHINSON (10).

Notre appareil peut produire avec le milieu de STERNE 150.000 doses vaccinales * par jour en culture continue, avec un débit moyen de 5 litres/heure de culture dense, lorsqu'il fonctionne à plein ; 3.000 boîtes de Roux seraient nécessaires pour obtenir cette même récolte.

Les germes obtenus ne montrent aucun signe de dissociation et l'antimousse utilisé respecte infégralement l'élaboration de l'antigène capsulaire, ce qui est une garantie très sérieuse de la qualité du vaccin.

II nous faut ajouter ici que des essais de culture de Solmonella et de Brucella ont été tentés avec ce même fermentateur et que nous avons obtenus des résultats très encourageants avec les Salmonella.

$$
\begin{aligned}
& \text { Institut d'élevage } \\
& \text { et de médecine vétérinaire } \\
& \text { des pays tropicaux. }
\end{aligned}
$$

\section{REMERCIEMENTS}

Nous tenons à remercier tout particulièrement notre confrère $M$. DOUTRE, dont l'aide nous fut précieuse au cours de ces essais.

* NB. Dose vaccinale : $2 \mathrm{mg}$ de poids sec de bactéries par bovin. 


\section{SUMMARY}

Heavy growth culture of Pasteurella 'multocida as the method of choice for the production of a vaccine against bovine pasteurellosis

The author, using an ordinary fermentation agent for the concentrated culture of $P$. multocida in Sterne's medium, was able to obtain a production of 150,000 vaccinal doses ( $2 \mathrm{mg}$ dry weight of bacteria per dose) per day in continuous culture using an average of 5 litres/hours of heavy medium whereas 3,000 Roux flasks would be necessary to harvest the same quantity. The organisms obtained show no sign of dissociation and the foam preventing agent used ensures the production of the capsular antigen which is an important factor in the quality of the vaccine.

\section{RESUMEN}

El cultivo denso de Pasteurella mulfocida, méthodo de elección para la producción de la vacuna contra la Pasteurelosis bovina.

El autor, utilizando un fermantador no especializado para el cultivo denso de $P$. multocida en medio de Sterne, obtiene una producción de 150.000 dosis vacunales al día $(2 \mathrm{mg}$ de peso seco de bacteria por dosis) en cultivo continuo, con un consumo medio de 5 litros/hora de cultivo denso; para obtener esta misma cosecha, serian necessarias 3.000 cajas de Roux.

Los gérmenes obtenidos no mestran ninguna señal de disociaciôn y el antiespuma utilizado respeta integralmente la elaboración del antigeno capsular, lo cual es una muy seria garantia de la calidad de la vacuna.

\section{BIBLIOGRAPHIE}

1. BAIN (R. V. S.). - Haemorrhagic septicaemia of cattle. Observations on some recent work. Brit. vet. J., 1959, II5 (10): 365-9.

2. BAIN (R, V. S.) et JONES (R. F.). - The production of dense cultures of Pasteurella multocida. Brit, vet. J., 1958, II4:215-20.

3. CARTER (G. R.). - Studies on « Pasteurella multocida ». I. A hemagglutination test for the identification of serological types. Amer J. vet. Res., 1955, $16: 481-4$.

4. CHAIN (E. B.), PALADINO (D.), CALLOW (D. S.), UGOLINI (F.) et VAN DER SLUIS (J.). - 1952, Org. mond. Santć, Rapp. tech. no 10,$6 ; 73-97$.

5. MONOD (J.). - La technique de culture continue ; théorie et applications. Ann. Inst. Pasteur, 1950, $79:$ 390-410.

6. NEWING (C. R.) et MAC LEOD (A. K.). Magnetically induced vortex for small-scale aerated culture studies. Nature (London), 1956, 177 (4516) : 939-40.

7. NOVICK (A.), et SZILARD (L.). - Description of the chemosiat. Science, 1950, 112: 715-6.

8. Rapport de la Réunion F. A. O. sur la septicémie hémorragique. Manille, Philippines
(30 novembre-5 décembre 1959). Recommandations.

9. STERNE (M.). - The growth of Brucella abortus strain 19 in aerated dialysed media. J. gen. Micr., 1958, 18 (3) : 747-50.

10. STERNE (M.) et HUTCHINSON (I.). - The production of bovine haemorrhagic septicaemia vaccine by continuous culture. Brit. vet. J., 1958, II4:176-9.

11. STERNE (M.). - Réunion F. A. O. sur la septicémie hémorragique, Manille, Philippines (30 novembre-5 décembre 1959). Docu- ment de travail no 4.

12. VAN DRIMMELEN (G. C.). - Strain 19 Brucella vaccine. I. Production of vaccine by the shake flask technique. Onderstepoort J. vet. Res., 1956, 27 (2) : 205-14.

13. VAN DRIMMELEN( G. C.). - Strain 19 Brucella vaccine. $V$. Mass production in the Brucella vortex aerated culture apparatus. Onderstepoort J. vet. Res., 1958, 27 (4) : 539-47.

14. WEYLAND $(H$.$) . - The continuous flow$ culture of micro-organisms as a research tool in biology and biochemistry. J. South Afr. Med. Assoc., 1960, 31 (1) : 7-13. 